\title{
RFID-enabled complex event processing application framework for manufacturing
}

\section{Ji Fang*, George Q. Huang and Ting Qu}

Department of Industrial and Manufacturing Systems Engineering,

The University of Hong Kong,

Pok Fu Lam Road, Hong Kong, China

Email: fangji@hku.hk

Email: gqhuang@hku.hk

Email: quting@hku.hk

*Corresponding author

\section{YingFeng Zhang}

Key Laboratory of Contemporary Design and Integrated

Manufacturing Technology,

Ministry of Education,

Northwestern Polytechnical University,

Xi' an, 710072, China

Email: zhangyf@nwpu.edu.cn

\begin{abstract}
In order to face up with classic manufacturing challenges such as high work in progress (WIP) inventories, complexity in production planning and scheduling, and low labour and machine utilisation, many manufacturing companies made their efforts in implementing RFID (Radio Frequency Identification Devices) throughout the manufacturing workshops. Through this way, all production data in manufacturing fields can be obtained in real time, and it improves the flexibility and responsivity to the changing market for the companies. However, at the same time the RFID deployment also introduces a new challenge which requires an effective and efficient method to handle the large amounts of events. This paper proposes an application framework for a real-time Complex Event Management System (CEMS) based on RFID equipments deployment. With the use of Complex Event Processing (CEP) technologies, this system allows users to obtain interested and meaningful information from large numbers of primitive events captured from the RFID devices deployed in manufacturing shop-floor in real time. This paper presents the RFID deployment infrastructure first, and then system design of the CEMS is proposed.
\end{abstract}

Keywords: RFID; Auto-ID; complex event processing; data capturing; realtime manufacturing; production planning and scheduling; Petri net.

Reference to this paper should be made as follows: Fang, J., Huang, G.Q., $\mathrm{Qu}, \mathrm{T}$. and Zhang, Y.F. (20xx) 'RFID-enabled complex event processing application framework for manufacturing', Int. J. Services Operations and Informatics, Vol. x, No. y, pp.xx-xx.

Biographical notes: Ji Fang is a PhD student in Department of Industrial and Manufacturing Systems Engineering, The University of Hong Kong. His research interests include computer integrated manufacturing, product and production service systems, workflow and process management and complex event processing. 


\section{J. Fang et al.}

George Q. Huang is a Professor in Department of Industrial and Manufacturing Systems Engineering, The University of Hong Kong. He gained his BEng and $\mathrm{PhD}$ in Mechanical Engineering from Southeast University (China) and Cardiff University (UK) in 1983 and 1991 respectively. He is a Chartered Engineer (CEng), a member of IEE (UK), HKIE (HK), ASME (USA), and IIE (USA).

Ting $\mathrm{Qu}$ is a Postdoctoral Research Fellow working in the Department of Industrial Manufacturing and Systems Engineering at the University of Hong Kong, China. His research interests include computer integrated manufacturing, product and production service systems, and supply chain management.

Ying-Feng Zhang is Professor of the Key Laboratory of Contemporary Design and Integrated Manufacturing Technology at Northwestern Polytechnical University, China. He received his PHD degree in Mechanical Engineering from Xi'an Jiaotong University, China in 2005. Currently, His teaching and research interests are in the area of networked manufacturing, intelligent manufacturing, e-manufacturing, RFID technologies. He has published a number of articles in journals such as the Journal of RCIM, Journal of AMT etc.

\section{Introduction}

Radio frequency identification is a powerful Auto-ID technology that allows people to achieve business processes and real-time data visibility. Compared to traditional Auto-ID technology, such as barcode, RFID tags can be read without line-of-sight scanning and any physical contact for readers to retrieve the information contained. RFID readers are also able to detect and read multiple tags simultaneously. As such, RFID is regarded as a new generation of Auto-ID technology (Derakhshan et al., 2007). It has been widely applied in manufacturing (Liu et al., 2004; Huang et al., 2007; Huang et al., 2008a; Huang et al., 2008b), supply chain management (Michael and McCathie, 2005; Delen et al., 2007) and other related industries involving real-time object tracking purpose, such as asset tracking (Bhanage et al., 2007; Patil et al., 2008), drug safety and healthcare (Wu et al., 2005; Hakim et al., 2006; Wang et al., 2006b), etc.

With the application of RFID solutions in the mentioned fields, a new challenge is also introduced. That is how to handle the huge volume of information and events generated from the deployment fields, especially when real-time response is a requirement. In this paper, we will focus on the RFID manufacturing applications which often contain large number of data collection points with RFID devices in fields. The events produced by RFID devices from manufacturing fields are called primitive events which are obtained directly from hardware level through invoking the corresponding device drivers or SDKs. The RFID technology provides a feasible solution for capturing the real-time production data from manufacturing shop-floors and brings the data visibility for the manufacturing environments. However, the primitive events captured from RFID devices on manufacturing fields are not meaningful enough to be directly used for business and execution level due to the lack of an effective event and data management system to link up the isolated information. Specifically, the lack of time, spatial and causal relationships (Luckham and Brian, 1998; Derakhshan et al., 2007) among primitive events makes it difficult for the existing information systems such as 


\section{RFID-enabled complex event processing}

ERP (Enterprise Resource Planning), SCM (Supply Chain Management), CRM (Customer Relationship Management), MES (Manufacturing Execution System), etc. to obtain really meaningful high level and actionable information. Take products packing as an example, only when a primitive event capturing the productions assembly finished is combined with another event representing container or packing box ready and sealed, a meaningful event reflecting packing finished and ready for transportation event could be generated. Otherwise, either event is not enough to trigger new event for transportation.

The enormous primitive events generated from RFID devices usually only carry an identity code (EPC) for the tagged object, captured time and perhaps the location information when the deployed tag is not sophisticated. Therefore, the primitive events are often too low level and too simple for application layer usage. Meanwhile, the misreading detections and cumulatively reading in manufacturing shop-floors are also issues needed to be considered. Furthermore, currently many enterprises apply workflows technology to manage their business processes or production flows. However, it is still a problem how to efficiently and effectively integrate the RFID deployment and the defined workflows.

To meet the challenges mentioned above, Complex Event Processing (CEP) technology is a good candidate. Complex event processing (Luckham and Brian, 1998) is a kind of technology and method that helps us to obtain more meaningful and actionable information from large amount of low-level or primitive events and to control the eventdriven information systems. The primitive events are processed in several different ways including filter, aggregation. The unwatched events are directly omitted. Primitive events which are matched to the pre-defined pattern are collected and aggregated into a new complex event. For those repetitive primitive events, they are counted and transformed into a new single event with the exact number of occurrence. Through these ways, the primitive events are combined to the time, spatial and causal relationships among themselves. More meaningful information is then discovered. CEP is a relatively new technology, and researchers have proposed lots of different architecture of CEP solutions since 1990s. Luckham and Brian (1998) present an overview on CEP concepts and provide a demonstration on how to apply these techniques to some specific distributed information systems. Perrochon et al. (1999) report the foundations and aims of CEP in detail and then propose an agent-based complex event processing network. Wang et al. (2006a) suggest a rule-based approach to implement automatic RFID data transformation between the physical world and the virtual world, and give out the detail event classification and event aggregation based on the defined semantic space.

In recent research, CEP technology is widely used in many types of applications from different fields, including manufacturing, enterprise business application, logistics management system, security, etc. Dong et al. (2006) discuss the design of RFID middleware system based on CEP with manufacturing scenarios. Kim et al. (2006) propose a RFID middleware framework for processing logistics information with contextual event assistant. Zang and Fan (2007) apply CEP technology to enterprise information systems, and demonstrate how the event processing engine works and interacts with other existing information systems, including ERP, SCM and CRM. Especially, Zang and Fan extract complex event pattern from workflow model in their proposed system, which makes CEP more cooperative. IBM (Collins, 2008) and Siemens (Holloway, 2008) also have developed solutions for enterprise information processing based on CEP technology. 


\section{J. Fang et al.}

CEP technology is discussed a lot in recent research, but there is still very limited work conducted for detail implementation for an overall RFID-based complex event processing system framework. Current existing complex event representation is mostly performed in text-based semantic space. Actually, graphical complex event pattern representation and modification are more intuitionistic, easy to understand and closely connected to the physical meaning in real world. In this paper, a re-usable infrastructure of complex event management system is developed for event-driven systems. This paper is structured as follows. Section 2 presents the RFID deployment, complex event concept and representation model for manufacturing environments. Section 3 discusses the complex event management system infrastructure and its detail design. Section 4 provides a simple example for the usage of the proposed framework. Future work and conclusion are discussed in Section 5.

\section{RFID deployment and complex event in manufacturing}

In this section, a RFID deployment infrastructure will be illustrated first. Then, the complex event in manufacturing environment is discussed on its definition and representation model.

Figure 1 shows the RFID deployment in a hierarchical structure. The lowest level is the manufacturing fields where monitoring objects RFID tagged are. A manufacturing factory may consist of one or more shop-floor production lines. In every production line, there are several workcells each of which in turn has a variety of manufacturing objects, such as operators, machines, materials, etc. Different production lines are often corresponding to different production processes. The second level is the RFID readers. Different kinds of readers are responsible for different manufacturing environments. For example, the staff cards in a factory are often HF (high frequency) RFID cards. In order to reduce the checking time at warehouse entrance, UHF (ultra high frequency) RFID cards are usually applied.

The third layer is the work centre gateway. This layer is to integrate and drive all different types of readers through a designed gateway which supports 'plug and play' for more than five different types and brands most common readers on the market, such Alien, Motorola, ACS and etc. It also provides a suite of software applications for managing operations and activities of RFID devices. Work centre Gateway captures realtime manufacturing information and routes data to upper-level applications or information systems according to a pre-defined workflow to enable appropriate real-time manufacturing processes. In the proposed system, the work centre gateway makes two innovative contributions. The first contribution is 'Plug and Play' scalability. RFID devices can be plugged in or removed from the work centre gateway without stopping their functions for any engineering change. The second one is 'reconfigurable', which means RFID devices can be easily configured for different processes with varying real-time information capturing requirements.

The fourth layer is the shop-floor gateway which is responsible for the networking among all the work centre gateways and to provide a two-way communication channel between shop-floor and upper level enterprise applications. From operations to enterprise decisions, shop-floor gateway captures real-time information (e.g. real time work-inprogress production data) from the associated work centre gateways and converts the information into standard formats (XML) to be directly used by enterprise application 


\section{RFID-enabled complex event processing}

systems (EASs). From decisions to operations, on the other hand, shop-floor gateway can receive enterprise's decisions, such as production planning and scheduling, and translate them into production orders or tasks that can be readily used by shop-floor operators or devices. The fifth layer is the existing enterprise information management systems which can benefit from the real-time data collection from this RFID deployment. The top level is the user level. Users can acquire the interested information which is processed and transferred from shop-floor gateway.

Figure 1 RFID deployment (see online version for colours)

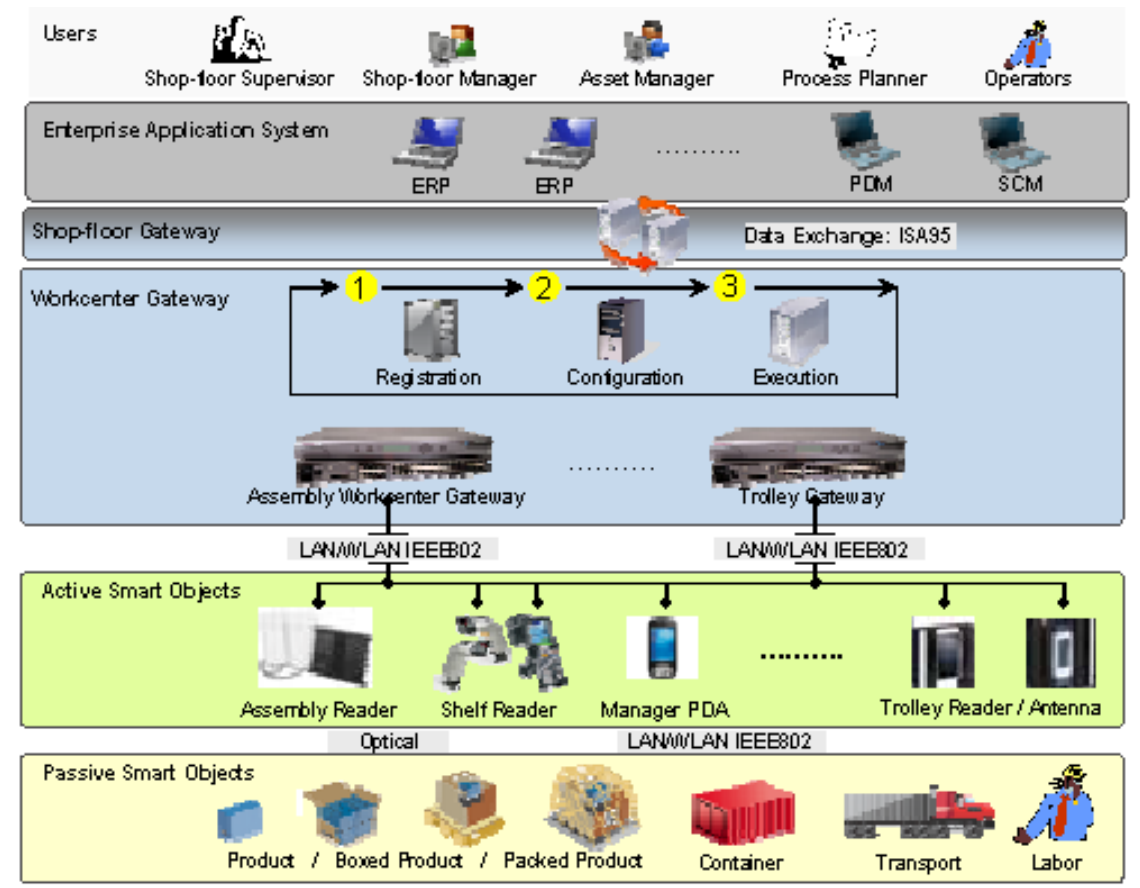

\subsection{Complex event}

Events are defined as objects which are records of activities in system (Luckham, 2002). In The Application Level Events (ALE) Specification of EPC global standard (EPCglobal, 2009), the processing actions and functions implied by the reading activities and writing activities are defined as events. In real world, event can be a user to log on with a RFID card; materials to enter warehouse; products to be put onto shelf, etc.

A complex event is an aggregation of series of other events (Luckham, 2002). Events are aggregated according to the pre-defined pattern which contains the information of the relationship among them. This kind of aggregated composite event is defined as complex event. For its physical meaning, it can be a user logs on with a RFID card and puts some products onto a shelf. Then, a complex event is generated to represent that a user putting products onto shelf. At the same time a notification is also sent to users that products are ready for transportation. 


\section{J. Fang et al.}

In this paper, single events generated directly from manufacturing sites are called primitive events. For example, a reading activity of a RFID tag on pallet is regarded as a primitive event in the proposed system. Complex event consists of a series of primitive events organised in a pre-defined pattern to represent a business workflow or work process.

\subsection{Representation model}

Petri net is a great tool to establish model and analyse distributed processes for business, manufacturing and also IT sector (Taylor et al., 2007). Petri net has been widely used to represent workflow in recent research. Zang and Fan (2007) extract necessary complex event information from workflows which is represented by Petri net.

To better cooperate with the existing information systems (ERP, MES, etc.) and compatible with business workflows running in companies and enterprises, we apply Petri net to represent complex events in our proposed system, which also makes the defined complex events easier to be understood and maintained. The complex events are also described and saved as XML files for better universality. An editor with friendly user interface for workflows composition is provided in the proposed system.

Figure 2 shows an example on Petri net representation for a complex event of pharmaceutical production quality detection. The circles which represent places are event triggering points here, where the events are collected. The transitions and connections remain the same with that for workflows. The detail data model for complex events will be discussed in our next paper.

Figure 2 Example of Petri net representation (see online version for colours)

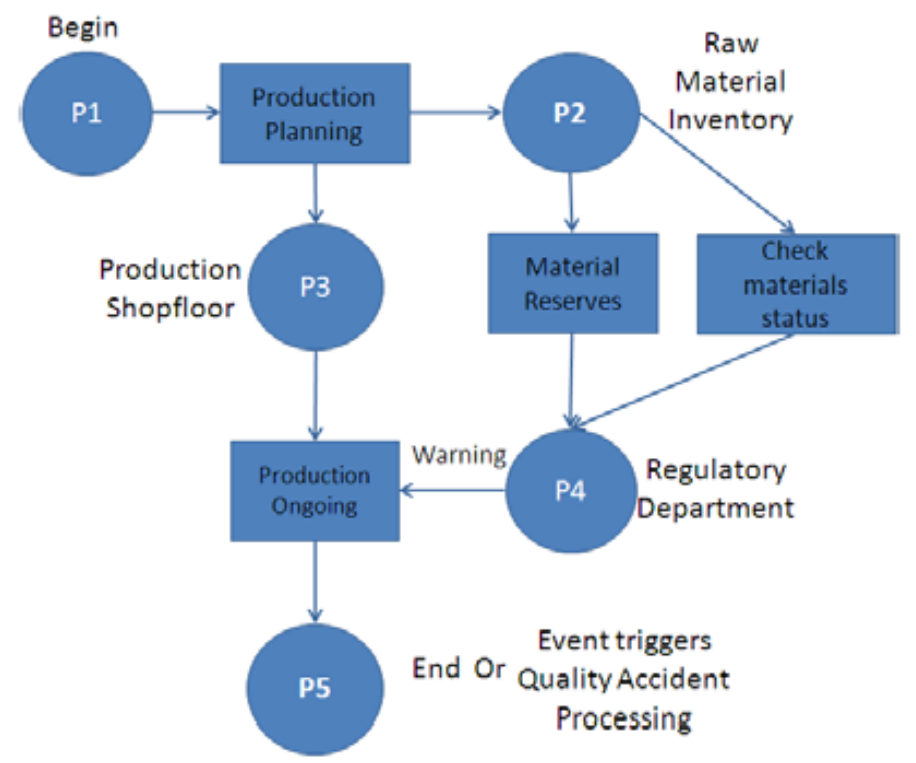




\section{RFID-enabled complex event processing}

\section{Complex Event Management System (CEMS)}

As discussed in the introduction section, the CEMS needs to fulfil the following four functions. Firstly, CEMS need to process and organise primitive events based on pre-defined patterns. Secondly, it needs to analyse the matched complex event patterns and report to other enterprise application systems. Thirdly, it should be re-configurable to meet different requirements or engineering changes. Fourthly, the CEMS provides interfaces for users to do track and trace operations. The role of CEMS in a RFIDenabled manufacturing environment is described first in this section, and then the detail system structure and key components of the CEMS are discussed.

\subsection{Role of CEMS}

The CEMS works between the shop-floor gateway and the existing enterprise applications, such as ERP, MES and the SCM systems. The application of this system will enable manufacturing enterprise to implement distributed or complex workflow tracking and gain more accurate track and trace on product material and work in progress inventory level. It also improves enterprise's ability of potential issues warning and detection, and enhances the performance on resources planning. The role of this system is shown in Figure 3.

Figure 3 Roles of CEMS (see online version for colours)

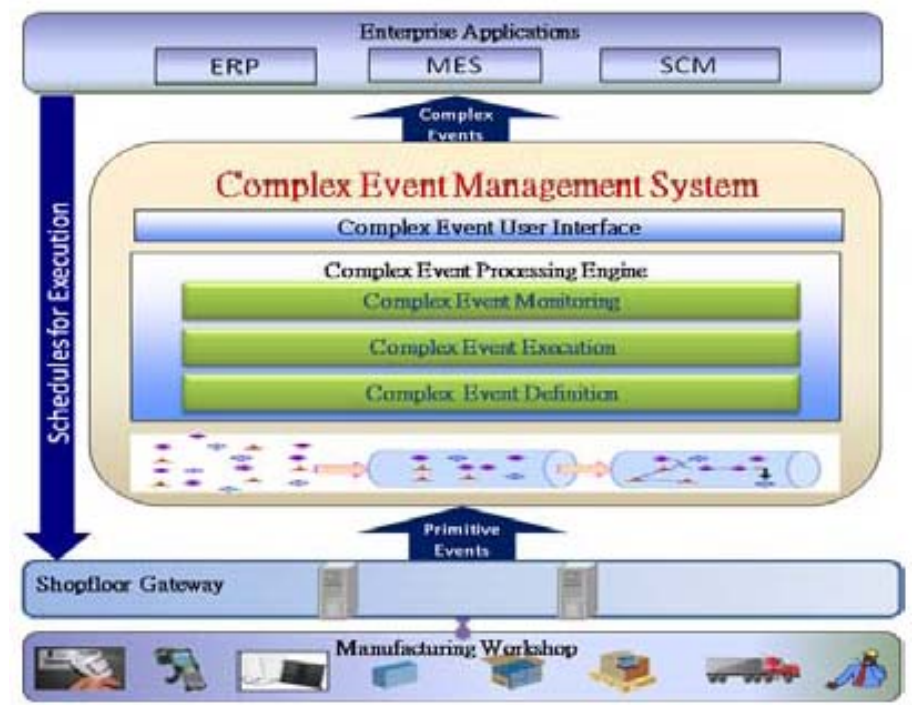

The primitive events are detected and collected by work centre gateways and then submitted to the shop-floor gateway. All the primitive events are aggregated and integrated into high-level complex events by the CEMS. Complex event report will be generated and sent to the other enterprise applications for the support of decision making. 


\section{J. Fang et al.}

\subsection{Infrastructure of CEMS}

The proposed infrastructure is consistent with the manufacturing hierarchy. A manufacturing factory hosts one or more shop-floor production lines, and each production line consists of workcells which are involved of a variety of manufacturing objects such as operators, machines, materials, etc. Different production lines are often designed to enable different production processes. Primitive events are generated from shop-floor production lines and submitted to shop-floor gateway. Interested events are transferred to CEMS for processing from the shop-floor gateway. The CEMS will automatically aggregate and detect complex events which are pre-defined in Petri nets from primitive events, and generates reports to other enterprise applications systems in upper level after analysis. The most important component of this system is complex event processing engine which is responsible for complex event definition, complex event execution and complex event monitoring. The detail components of this engine are discussed in next sub-session.

As shown in Figure 4, complex event user interface provides friendly both text-based and graph-based methods for users to proceed inquiries or monitoring operations. It is also the bridge to communicate with other application systems in upper level. The complex event processing engine is the core of this proposed system. It is composed by three parts, complex event definition tool, complex event executer and complex event monitor. Event registry and repository are databases for this proposed system. Registry is for storing event types and descriptions. The dynamic data generated by events is stored in the event repository.

Figure 4 CEMS infrastructure (see online version for colours)

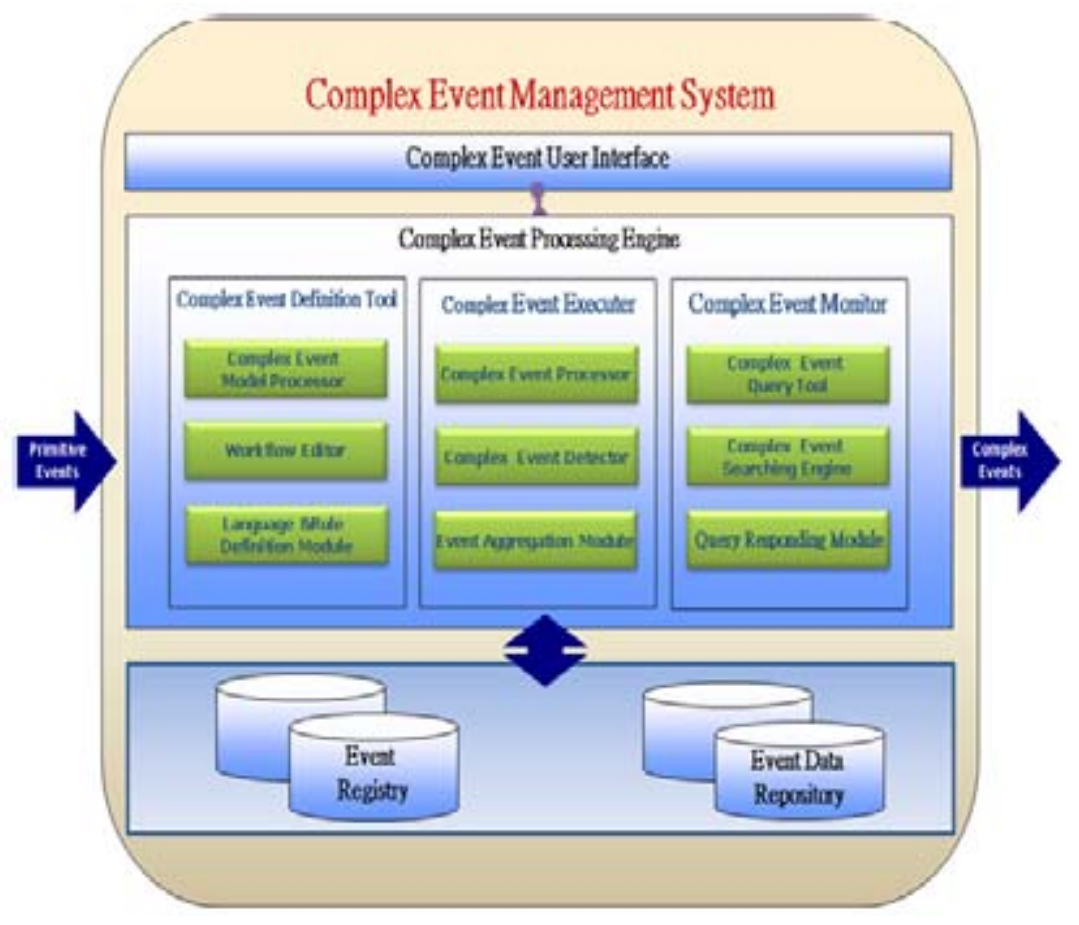




\section{RFID-enabled complex event processing}

Complex event patterns are defined through the complex event definition tool with either textbased or graph-based interface. When the primitive events are submitted from the shopfloor gateway, the interested or monitored events are detected and aggregated into complex event based on the defined patterns by the complex event executer. Track and trace ability are implemented with complex event monitor which can also generate kinds of query reports for ERP, MES and other enterprise applications.

\subsection{Key components of CEP engine}

As shown in Figure 4, there are three modules in this engine, complex event definition tool, complex event executer and complex event monitor. They are discussed respectively in the following sections.

\subsubsection{Complex event definition tool}

The complex event definition tool is to create new complex event types or patterns by composing corresponding workflows through a provided user interface. Besides being responsible for the complex events definition, it also provides interface to reconfigure and maintain the defined workflows and complex event patterns. The workflows and complex events are also described in a unified model as XML files. This module is a complex event type registration and maintenance tool. It is composed by three subcomponents, language and rule definition module, workflow editor and complex event model processor. The architecture of this module is shown as Figure 5.

Figure 5 Architecture of complex event definition tool (see online version for colours)

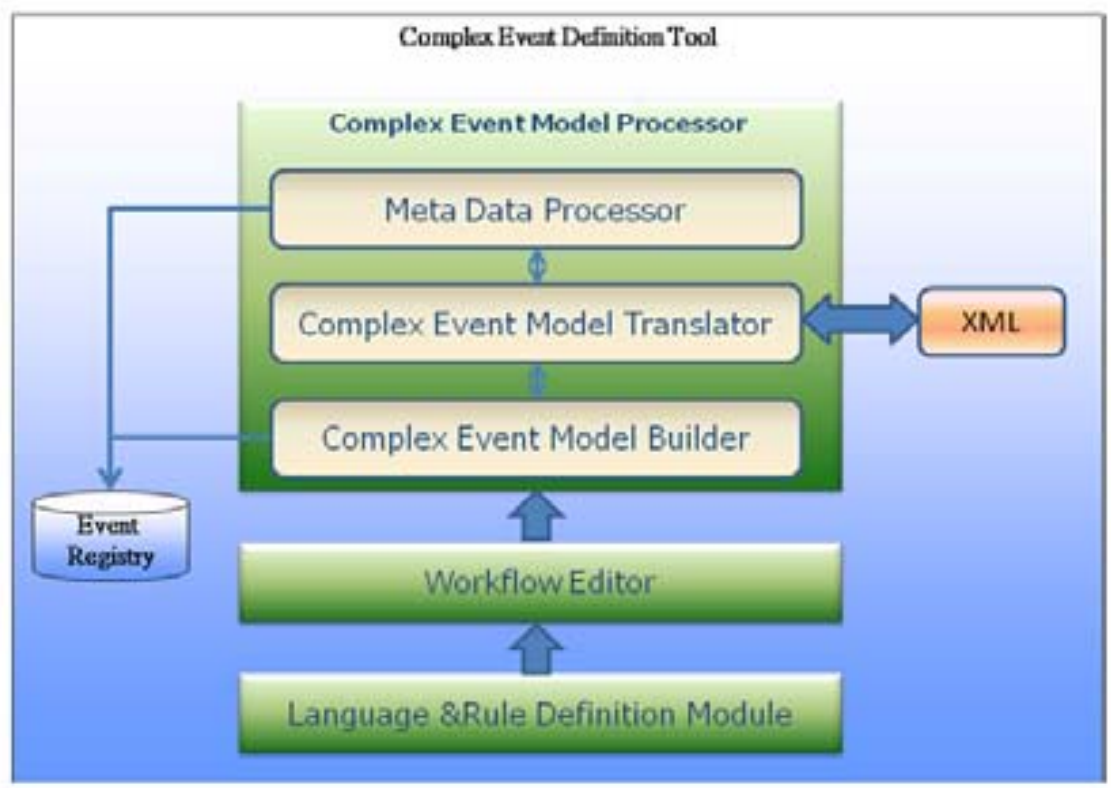




\section{J. Fang et al.}

Language and rule definition module is responsible for basic semantic operator definition for representing the relationship among primitive events in a complex event pattern. The defined operators will be used by the workflow editor.

Workflow editor is a graph-based tool to define workflow. It allows user to compose workflow with simple draw and drop operations.

Language and rule definition module enables users to define and maintain the text expression method and rules for complex events.

Complex event model processor is responsible for building complex event models from defined workflows through sub-module complex event model builder; also transforming the models into XML file through sub-module complex event model translator; and saving these meta information into event repository through sub-module metadata processor.

\subsubsection{Complex event executer}

The complex event executer implements complex events aggregation and detection in real time. The processing and analysis of the complex events are completed in this module. The consequence report of the complex events will be submitted to the complex event user interface and sent to other application systems in the upper level and regulatory service systems. This component is consisted by two major sub-modules, complex event detector and complex event processor. Its architecture is shown as in Figure 6.

Figure 6 Architecture of complex event executer (see online version for colours)

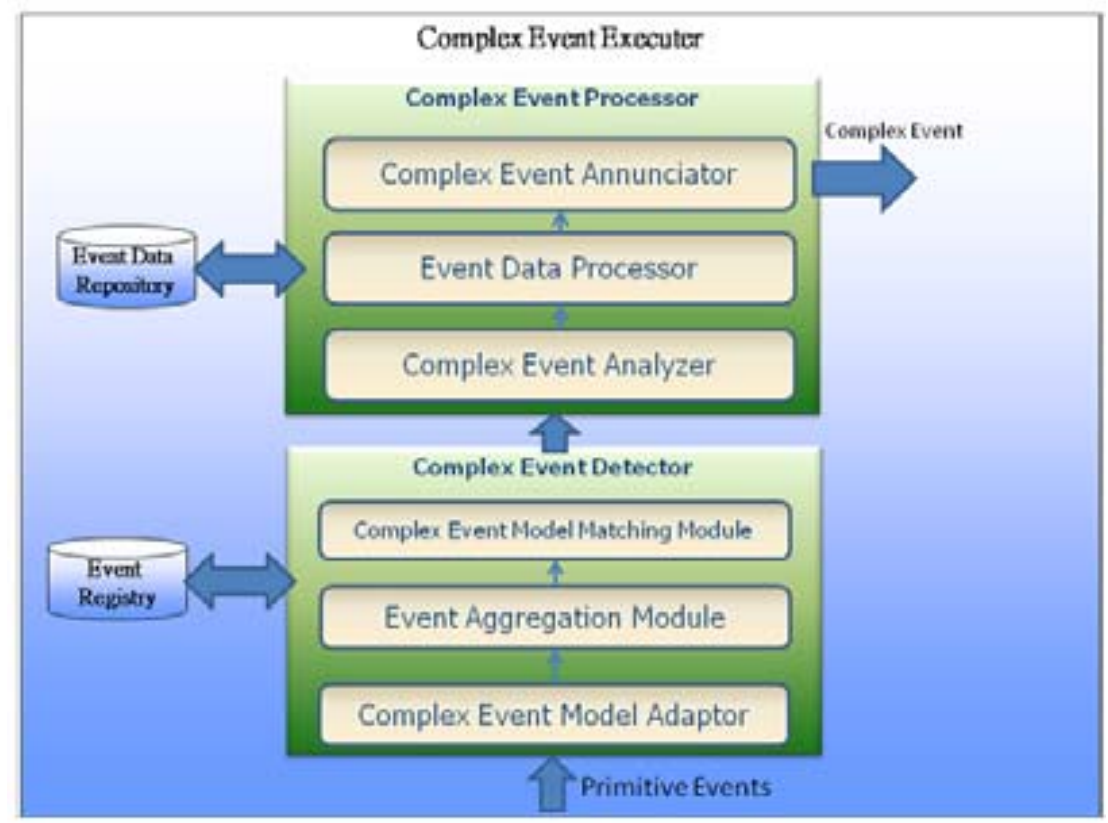




\section{RFID-enabled complex event processing}

The complex event detector will classify the received primitive events based on the meta information (Complex Event Model Adaptor), then aggregate the primitive events (Event Aggregation Module) and match to the defined complex event patterns (Complex Event Model Matching Module). The Complex Event Processor includes three sub-modules which are Complex Event Analyser, Event Data Processor and Complex Event Annunciator. Complex Event Analyser analyses the received complex event and generates consequence result which includes abstract information and event data. Event Data Processor saves all generated event data into database. Complex Event Annunciator generates reports for other enterprise information systems.

\subsubsection{Complex event monitor}

Complex event monitor provides a graph-based user interface to track the defined and activated workflow. The progress of the workflow under monitoring is tracked through the complex event executer. This module updates the status and shows it on the graph in real-time. The architecture of this component is shown as Figure 7.

The incoming inquiry from complex event user interface is translated into complex event semantic expression (Complex Event Model Translator) first, and then the engine will search the matched pattern in event repository. Corresponding action and report will be generated based on the inquiry types.

Figure 7 Architecture of complex event monitor (see online version for colours)

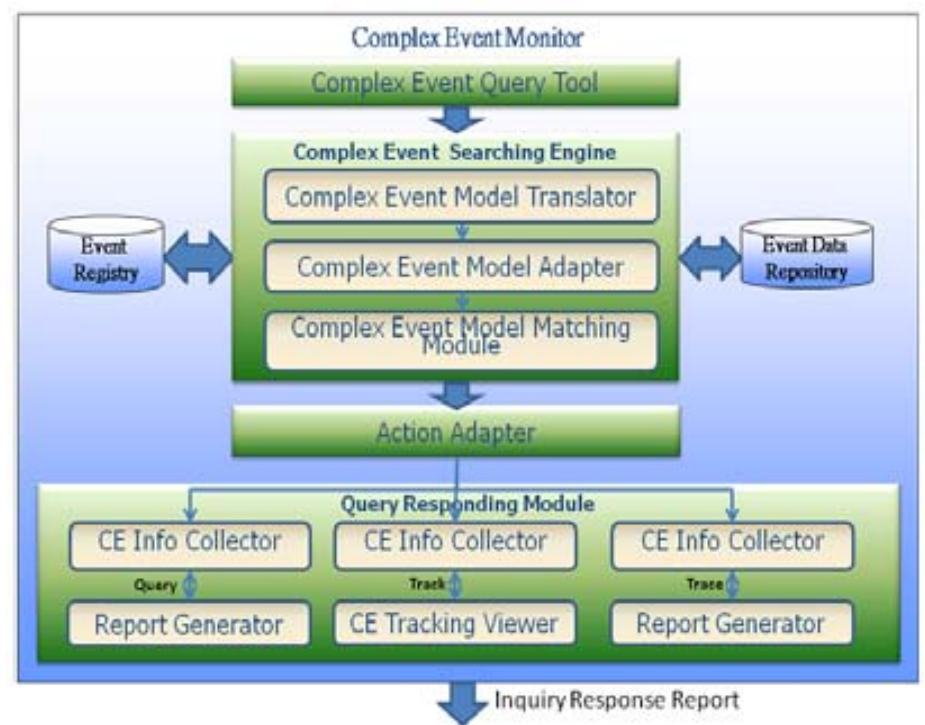

\section{Case study}

We take manufacturing plan and schedule as an example. Before this system, there are some problems between shop-floor production data and high-level enterprise systems as shown in Figure 8. 


\section{J. Fang et al.}

Figure 8 Problems between the two layers (see online version for colours)

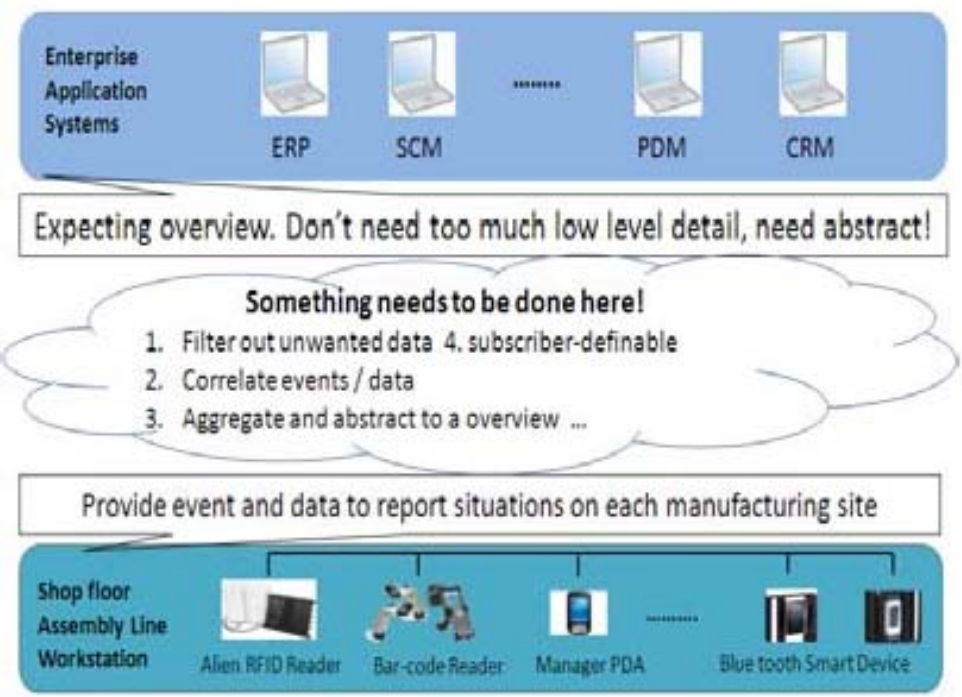

As show in Figure 3, the proposed system works between enterprise high-level application layer and shop-floor gateway. In this case, product manufacturing process consists of two major parts which are pre-assembly process and final assembly process. All the working stages for each processing step in this case are equipped with RFID devices. There are four processing steps in pre-assembly line, and two in final assembly line. The manufacturing process for this product in shop-floor is shown as Figure 9 below. The last step after assembly is product packing.

Figure 9 Manufacturing process in shop-floor (see online version for colours)

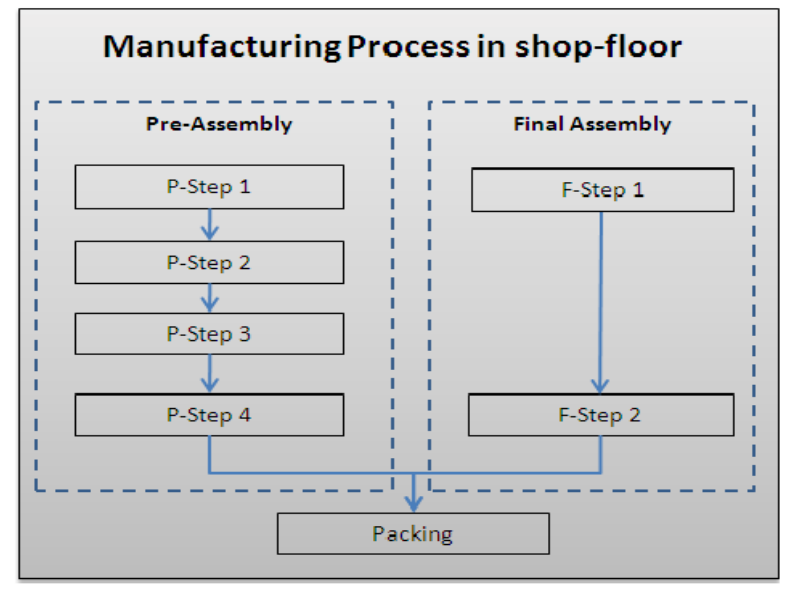

Manufacturing plan A for this product is defined as a high-level complex event which is composed by a primitive event which is product packing and two sub-complex events respectively corresponding to the pre-assembly process and final assembly process. With 


\section{RFID-enabled complex event processing}

the RFID devices, primitive events will be generated from shopfloor stages corresponding to the working steps. Therefore, the event structure corresponding to this manufacturing process is shown as Figure 10.

Figure 10 Corresponding event structure (see online version for colours)

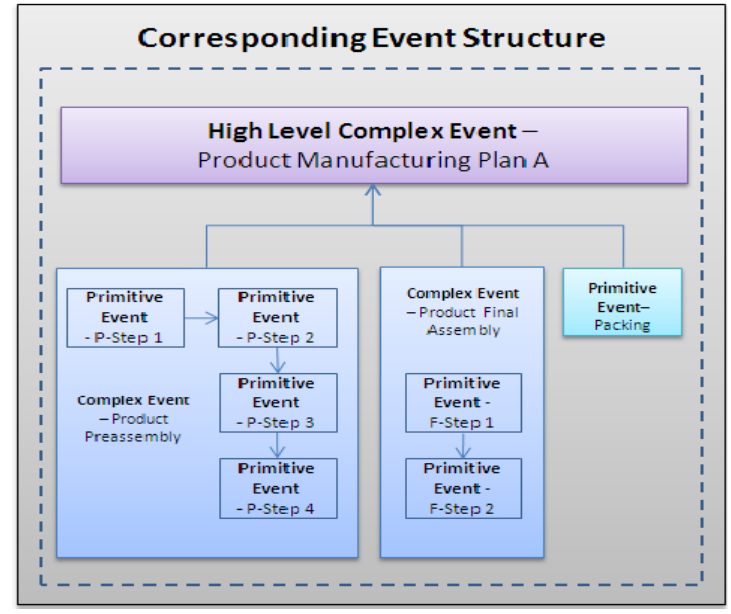

User can access event definition tool to compose event patterns for the mentioned events for this production plan execution mentioned above. This can be done through both text and graph-based method. The Petri net representation for this manufacturing plan $\mathrm{A}$ is shown as Figure 11. Actually, its structure is consistent with what is shown in Figure 10. The corresponding sub-complex event patterns are also included in this figure.

Figure 11 Event representation (see online version for colours)

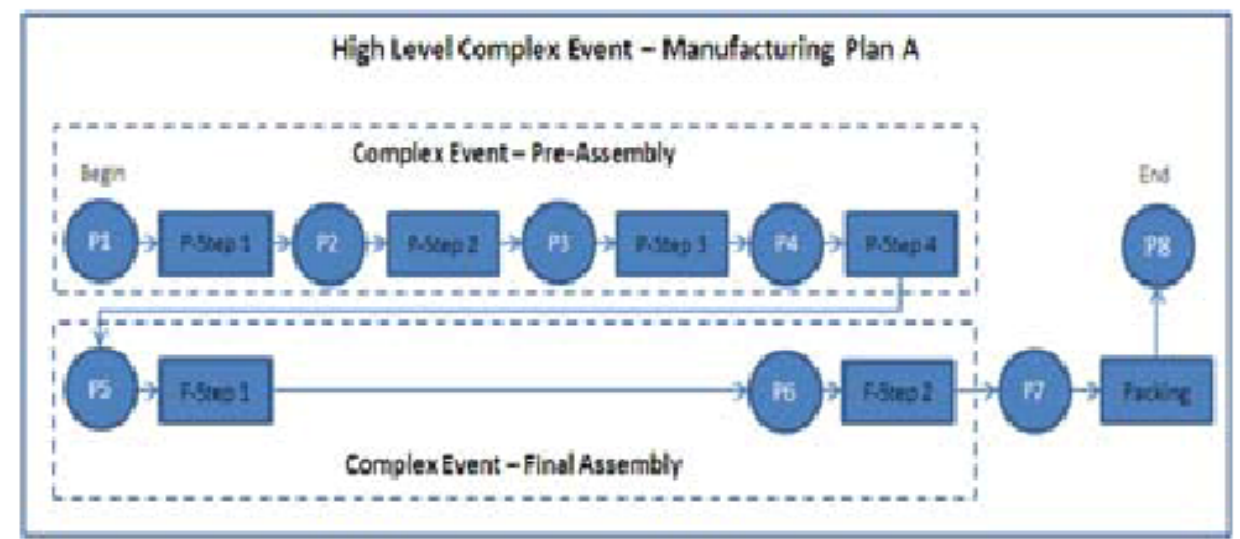

Complex event executor captures all triggered primitive events, and then updates the status of complex events accordingly. The schedule executing status can be obtained from the completion of each sub-complex event, so that the progress of manufacturing plan can be track in real time through complex event monitor. When exception happens 


\section{J. Fang et al.}

and affects the completion time of sub-complex event, the proposed system can trigger a warning message to enterprise systems, and then re-schedule can be generated if due date for manufacturing plan A is impacted. Events in different levels are linked up through event ID which is unique in database. These ID is generated once the event instance is established before execution. Figure 12 shows the reference structure of a linked data block for complex event product pre-assembly.

Figure 12 Data block reference structure (see online version for colours)

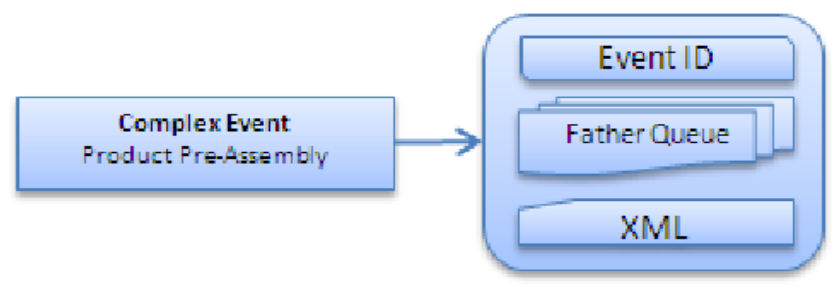

Father queue is a pool for storing upper level event ID. In this case, there is only one ID which is the one of event product manufacturing plan A. XML is used to save the detail description for this product pre-assembly event.

\section{Conclusion and future work}

In this paper, RFID deployment architecture for manufacturing environment is proposed first. Then, the concept of complex event for manufacturing is discussed. In order to handle large amount of events generated from shopfloor, the proposed CEMS is introduced. Two ways to define the complex event on both text and graphical representation for complex events are fulfilled in the proposed CEMS. A reconfigurable infrastructure of CEMS application model is proposed for manufacturing enterprise with detail designed modules. To facilitate easy data standardisation and communication, all the event data subscription and reporting are conducted in XML files. This CEMS is aim to improve the manufacturing performance, efficiency and accuracy for enterprises through detecting and aggregating the actionable and meaningful information from large number of low-level primitive events.

Further research is needed for the detail complex event execution, pattern matching algorithm and performance analysis for the proposed framework.

\section{References}

Bhanage, G.D., Zhang, Y., Zhang, Y., Trappe, W. and Howard, R.E. (2007) 'RollCall: the design for a low-cost and power efficient active RFID asset tracking system', The International Conference on 'Computer as a Tool'.

Collins, J. (2008) IBM launches RFID middleware. Available online at: http://www. rfidjournal.com/article/articleview/1291/1/1/ (accessed on 21 December 2008).

Delen, D., Hardgrave, B.C. and Sharda, R. (2007) 'RFID for better supply-chain management through enhanced information visibility', Production and Operations Management, Vol. 16, pp.613-624. 


\section{RFID-enabled complex event processing}

Derakhshan, R., Orlowska, M.E. and Xue, L. (2007) 'RFID data management: challenges and opportunities', 2007 IEEE International Conference on RFID, Gaylord Texan Resort, USA.

Dong, L.,Wang, D. and Sheng, H. (2006) 'Design of RFID middleware based on complex event processing', IEEE conference on Cybernetics and Intelligent Systems, 7-9 June, Bangkok, pp.1-6.

EPCglobal (2009) 'The Application Level Events (ALE) specification', EPCglobal Ratified Standard with Fixed Errata, Version 1.1.1.

Hakim, H., Renouf, R. and Enderle, J. (2006) 'Passive RFID asset monitoring system in hospital environments', Bioengineering Conference, pp.217-218.

Holloway, S. (2008) Siemens RFID middleware portfolio. Available online at: http://www. bloorresearch.com/analysis/10620/print2pdf.html (accessed on 21 December 2008).

Huang G.Q., Zhang, Y.F. and Jiang, P.Y. (2007) 'RFID-based wireless manufacturing for walkingworker assembly islands with fixed-position layouts', International Journal of Robotics and Computer Integrated Manufacture, Vol. 23, No. 4, pp.469-477.

Huang, G.Q., Zhang, Y.F. and Jiang, P.Y. (2008a) 'RFID-based wireless manufacturing for realtime management of job shop WIP inventories', International Journal of Advanced Manufacturing Technology, Vols. 7/8, No. 36, pp.752-764.

Huang, G.Q., Zhang, Y.F., Chen, X. and Newman, S.T. (2008b) 'RFID-enabled real-time wireless manufacturing for adaptive assembly planning and control', Journal of Intelligent Manufacturing, Vol. 19, pp.701-713.

Kim, Y., Moon, M. and Yeom, K. (2006) A Framework for Rapid Development of RFID Applications, Springer Berlin, Heidelberg, pp.226-235.

Liu, M.R., Zhang, Q.L., Ni, L.M. and Tseng, M.M. (2004) 'An RFID-based distributed control system for mass customization manufacturing', International Symposium on Parallel and Distributed Processing and Applications.

Luckham, D.C. (2002) The Power of Events, 1st ed., Addion-Wesley, Boston, pp.3-26, 87-126, $145-174$.

Luckham, D.C. and Brian, F. (1998) Complex Event Processing in Distributed Systems. USA: Program Analysis and Verification Group, Computer Systems Lab, Stanford University.

Michael, K. and McCathie, L. (2005) 'The pros and cons of RFID in supply chain management', International Conference on Mobile Business, pp.623-629.

Patil, A., Munson, J., Wood, D. and Cole, A. (2008) 'Bluebot: asset tracking via robotic location crawling', Computer Communications, Vol. 31, pp.1067-1077.

Penttilä, K. and Keskilammi, M. (2006) 'Radio frequency technology for automated manufacturing and logistics control. Part 2: RFID antenna utilisation in industrial applications', International Journal Advance Manufacturing Technology, Vol. 31, pp.116-124.

Perrochon, L., Stephane, K. and Luckham, D.C. (1999) Managing Event Processing Networks, USA: Computer Systems Laboratory, Departments of Electrical Engineering and Computer Science, Stanford University.

Taylor, I.J., Deelman, E., Gannon, D.B. and Shields, M. (2007) Workflows for e-Science, 1st ed., Springer, London, pp.190-207.

Wang, F., Liu, S., Liu, P. and Bai, Y. (2006a) Bridging Physical and Virtual Worlds: Complex Event Processing for RFID Data Streams, Springer Berlin, Heidelberg, pp.588-607.

Wang, S-W., Chen, W-H., Ong, C-S., Li, L. and Chuang, Y-W. (2006b) 'RFID applications in hospitals: a case study on a demonstration RFID project in a Taiwan hospital', Proceedings of the 39th Hawaii International Conference on System Sciences.

Wu, F., Kuo, F. and Liu, L-W. (2005) 'The application of RFID on drug safety of inpatient nursing healthcare', International Conference on Entertainment Computing.

Zang, C. and Fan, Y. (2007) 'Complex event processing in enterprise information systems based on RFID’, Enterprise Information System, Vol. 1, No. 1, pp.3-23. 